\title{
Clinic Pathological Study of Aneurysmal Fibrous Histiocytoma
}

\author{
Minna Gao, Xiao Lin* \\ Department of Pathology, Chongqing Medical University, Chongqing, China \\ Email:700525@cqmu.edu.cn, *linxiao@cqmu.edu.cn
}

How to cite this paper: Gao, M.N. and Lin, X. (2019) Clinic Pathological Study of Aneurysmal Fibrous Histiocytoma. Journal of Biosciences and Medicines, 7, 1-5. https://doi.org/10.4236/jbm.2019.75001

Received: March 11, 2019

Accepted: May 14, 2019

Published: May 17, 2019

\begin{abstract}
Aneurysmal fibrous histiocytoma (AFH) is a rare variant of benign fibrous histiocytoma (FH), Characterized by blood-filled spaces within the fibrohistiocytic tumor. AFH has a higher recurrence rate than $\mathrm{FH}$. The diagnosis of $\mathrm{AFH}$ is often problematic due to its overlapping morphological features with other skin tumors. The diagnosis of AFH depends on histological features and immunohistochemistry. The aim of this study is to understand the clinical and histopathological diagnostic criteria for AFH.
\end{abstract}

\section{Keywords}

Aneurysmal Fibrous Histiocytoma, Clinical Pathology, Diagnosis

\section{Introduction}

Aneurysmal fibrous histiocytoma (AFH) is a rare variant of fibrous histiocytoma (FH) with peculiar clinical presentation and pathological features. AFH was first reported by Santa Cruz in 1981 [1]. AFH is characterized by abundant capillaries and large blood-filled spaces. The main histopathological criterion for diagnosing this lesion is presence of blood-filled spaces simulating artifactual clefts or cavemous vascular channels within an ordinary FH. The clinical and morphological features of this entity are poorly recognized, thus resulting in diagnostic confusion. Here, we present the clinicopathological and immunohistochemical features of one case of AFH which was diagnosed at Department of Pathology in Chongqing Medical University and discuss the clinical pathological characteristics and distinguished point of AFH combined with review of cases in the literatures.

\section{Materials and Methods}

1) MATERIALS: One case of AFH was diagnosed at Pathological department 
in Chongqing Medical University.

2) METHODS: The formalin-fixed, paraffin-embedded tissue was sectioned at 5 um thickness for standard immunohistochemical staining. Slides in absolute ethanol, $2 \mathrm{~min}$ in 95\% ethanol, $2 \mathrm{~min}$ in $80 \%$ ethanol, and $5 \mathrm{~min}$ in distilled water, then rehydrated into distilled $\mathrm{H}_{2} \mathrm{O}_{2}$ through graded ethanol. Antigen retrieval was used to enhance Vimentin, FactorXIIIa, CD68, Bcl-2, CD99, CK, EMA, S100, Melan A, HMB45, CD34, STAT-6, and KI67 immunohistochemic by high press in citrate buffer ( $\mathrm{PH} \mathrm{6.0)}$ for $3 \mathrm{~min}$. Then the sections were washed and incubated those with 1 hour at room temperature. Slides were washed in phosphate-buffered saline, and then incubated with secondary antibody for $20 \mathrm{~min}$ at room temperature. After washed, slides were stained with $\mathrm{DAB}$ until desired stain intensity developed and mounted before observation by light microscopy.

\section{Results}

1) Clinical Findings: A 27 -year-old man was referred to our department for the evaluation of a tumor on the neck. The tumor had developed one year. Physical examination revealed a well-circumscribed brown mass on the neck, $1.0 \times 1.0 \times 1.0 \mathrm{~cm}$ in size. The mass was subject to excision biopsy.

2) Pathologic Findings: A little pile of grey-brown tissue whose diameter was $1.0 \mathrm{~cm}$ was present. On low-power microscopic: foam cells surrounded by hyalinized collagen bundles, which are typical features of lipidized FH. Proliferation of spindle-shaped fibroblasts presented some blood-filled spaces among. It showed large areas of hemorrhage surrounding stromal. Neither atypical nor necrosis was seen in this case [Figure 1]. Microscopic examination showed a resemblance to the original lesion, although it contained cystic, blood filled spaces. These spaces were lack an endothelial lining, being surrounded and lined by histiocytes, which contain hemosiderin, fibroblasts, and foam cells. Several cystic areas with no endothelial borders, but filled with red blood cells were seen. Higher magnifications: The solid intravascular growth was made up of massive proliferation of histolytic cells. Some of these cells showed abundant foamy

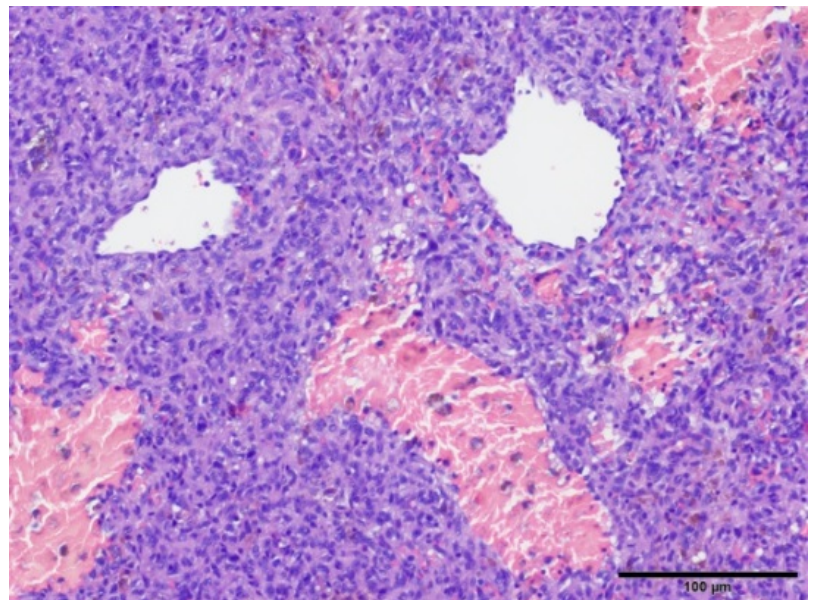

Figure 1. HE staining in AFH $(\times 200)$. 
cytoplasm. These were no cytological atypical or atypical mitosis. Other cells presented included scattered lymphocytes and eosinophils. Spindle cells were arranged storiform pattern was showed, the shape of cells ranging from oval to slender. Erythrocyte extravasation was found diffusely between the tumor cells. Multinucleate cells were observed. Besides the hemorrhagic areas, erythrocyte extravasation was found diffusely between the tumor cells. While, no endothelial lining was seen in areas of massive hemorrhage. Moreover, numerous compressed capillaries were observed. Immunohistochemistry: Tumor cells were positive for CD68 [Figure 2], FactorXIIIa [Figure 3], S100, Vimentin, and Desmin. Vascular markers CD34 was negative for the prominent, partially haemangiopericytoma-like vascularity in this case. The tumor cells were immunonegative for Melan A, HMB45, STAT6, CKpan and EMA.

\section{Discussion}

AFH is a rare variant of cutaneous fibrous histiocytoma that results from blood vessel proliferation and hemorrhage into fibrous hisliocytoma. AFH seem to

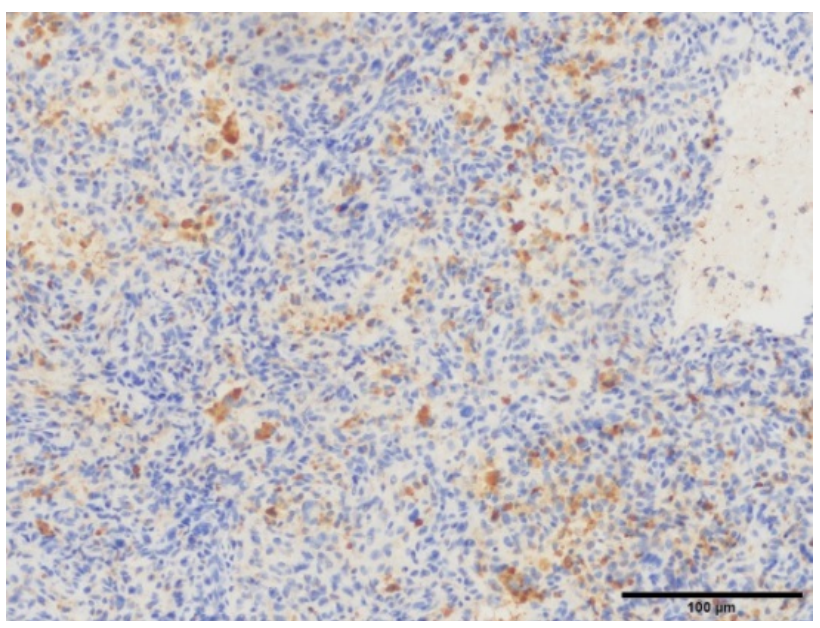

Figure 2. The positive staining of CD68 $(\times 200)$.

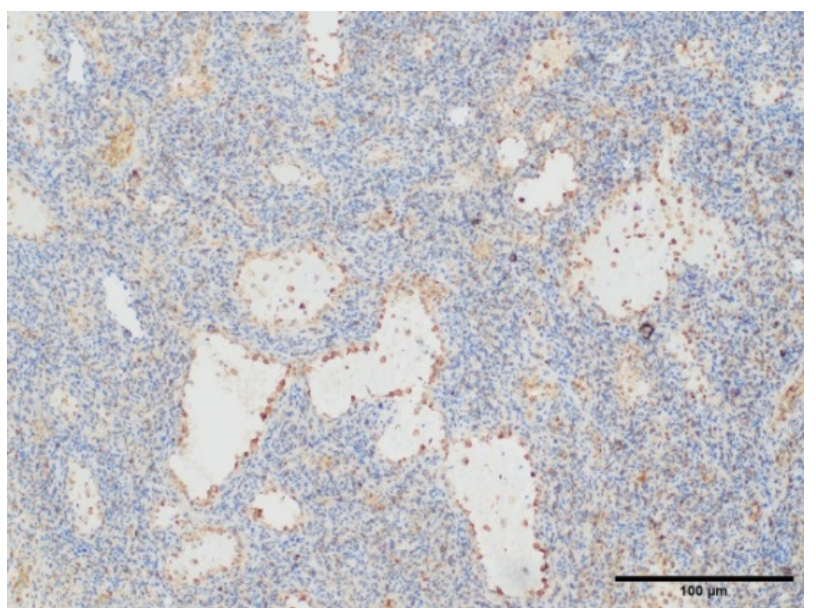

Figure 3. The positive staining of factor XIIIa $(\times 200)$. 
reflect the beginning of a process, characterizes by erythrocyte leakage, probably caused or at least favored by trauma. The pathogenesis of AFH is unknown. It may be caused by extra vacation of erythrocytes from capillaries leading to the cystic blood spaces. Some scholars indicated the pathogenesis of AFH may be connected with the immunosuppression and potential neoplastic disease [2] [3]. Loannis Panagoulos [4] reported the breakpoints on chromosomes 12 and 19 were between the ETV6 and PIK3C2G gene loci and proximal to the BCL3 gene in AFH by fluorescence in situ hybridization.AFH differs from ordinary benign fibrous histiocytoma by larger than average size, rapid growth due to intra-lesinalhaemorrhage and pigmentation [5]. In this case, the size of tumor was just $1.0 \times 1.0 \times 1.0 \mathrm{~cm}$, and the tumor did not grow rapidly. AFH most commonly affects children and young adults. The majority of AFH case occurs in the extremities, head, neck and trunk. AFH usually presents within the deep dermis and subcutaneous tissue. Symptoms of pain and tenderness are rarely encountered. But, there was a sudden increase in the size of the lesion owing to intraregional hemorrhage. Differential distinguish: 1: Kaposi's sarcoma: Koposi's sarcoma may be difficult to distinguish from AFH, but Kaposi's sarcoma is immunoreactive for CD34 and lack of fibrohistiocytic cells. Kaposi's sarcoma is a multifocal disorder with additional patch and plaque stages elsewhere. Histologically, the nodules after reveal, at their periphery, lymphangiomatoid and angiomatoid features characteristic of early patch and plaque lesions. Moreover, lesions are more irregularly arranged without epidermal hyperplasia and after contained numerous plasma cells. So, It is valuable to mark immunoreactivity for CD34, for differentiates AFH from Kaposi's sarcoma. 2: Angiomatoid malignant fibrous histiocytoma: It usually occurs in the deep subcutaneous tissue on the forear of children and young adults. Blood-filled cavity is surrounded by a storiform tumor with monomorphic, rounded, eosinophilia and giant cells.

Diagnosis: The main histopathologicalcriterion for AFH diagnosing is presence of blood filled spaces simulating artifactual clefts or cavernous vascular channels within an ordinary fibrous histiocytoma. It is important to recognizing histopathological examination for diagnosis of AFH. The expressions of factor XIIIa and CD68 were described differently in previous reports. Natheless, factorXIIIa and CD68 were displayed positively in our case, especially in the areas with hemosiderosis [6]. It is may be related to lysosome in cytoplasm. Some scholars indicate that diagnostic criteria for AFHare: 1) The tumor manifest as a dark brown or red noddle with diameter $<2 \mathrm{~cm}$, and no infiltration around it. 2) The histologic section shows the characteristic features of typical fibroids, accompanied by a large number of irregular hemorrhagic fissures, cystic structures and hemosiderin deposition. 3) Tumor cells express Vimentin and CD68. However, tumor cells show immunonegativity for vascular maker, such as CD34 and CD31.

Follow up and Treatment: AFH has a high local recurrence rate, because of incomplete first excision of large lesion. Higher recurrence rate and distance metastasis have also been described [7]. Recurrences have been reported in up to 
$19 \%$ of the cases following incomplete excision of AFH [8]. However, it is not termed "malignant" due to its benign microscopic appearance and favorable prognosis. The best treatment of AFH is local lesion excision. However, some scholars suggested extended resection for $\mathrm{AFH}$, because of its higher recurrence rate, regular follow-up is necessary.

In conclusion, $\mathrm{AFH}$ is a benign tumor, which is differentiated from Kaposi's sarcoma and Angiomatoid malignant fibrous histiocytoma by histopathology and immunohistochemistry. The confirmed diagnosis for AFH should depend on histopathology and immunohistochemistry. Surgical resection is the best treatment for $\mathrm{AFH}$.

\section{Conflicts of Interest}

The authors declare no conflicts of interest regarding the publication of this paper.

\section{References}

[1] Santa Cruz, D.J. and Kyriakos, M. (1981) Aneurysmal (Angiomatoid) Fibrous Histiocytoma of the Skin. Cancer, 47, 2053-2061.

https://doi.org/10.1002/1097-0142(19810415)47:8<2053::AID-CNCR2820470825>3. $\underline{0 . \mathrm{CO} ; 2-\mathrm{A}}$

[2] Calonie, E. and Fletcher, C.D. (1995) Aneurysmal Benign Fibrous Histiocytoma: Clinicopathological Analysis of 40 Cases of a Tumour Frequently Misdiagnosed as a Vascular Neoplasm. Histopathology, 26, 323-331. https://doi.org/10.1111/j.1365-2559.1995.tb00193.x

[3] Ichikawa, N., Kobayashi, M., Kimoto, M., Tanlkawa, A. and Tanaka, M. (2005) A Case of Multiple Aneurysmalfibrous Histiocytomas. Br J Demalol, 153, 664-665. https://doi.org/10.1111/j.1365-2133.2005.06773.x

[4] Panagopoulos, L., Gorunova, L., Bjerkehagen, B., Obmaier, I. and Heim, S. (2015) LAMTOR1-PRKCD and NUMA1-SFMBT1 Fusion Genes Identified by RNA Sequencing in Aneurysmal Benign Fibrous Histiocytoma with $\mathrm{t}(3 ; 11)(\mathrm{p} 21 ; \mathrm{q} 13)$. Cancer Genetics, 208, 545-551. https://doi.org/10.1016/j.cancergen.2015.07.007

[5] Xu, X.-L., Shao, X.-B., Chen, H., Wang, Q.-Q., Jiang, Y.-Q., Sun, J.-F. and Zeng, X.-S. (2012) Aneurysmal Fibrous Histiocytoma: A Clinical and Histopathologic Review of Five Cases. Chin J Dermatol, 45, 75-77.

[6] Kawakami, Y., Oyama, N., Nishibu, A., Nakamura, K. and Kaneko, F. (2006) A Case of "Giant" Aneurysmal Benign Fibrous Histiocytoma. ClinExpDermatol, 31, 456-457. https://doi.org/10.1111/j.1365-2230.2006.02074.x

[7] Shi, H., Li, H., Zhen, T., et al. (2015) Clinicopathological Features of Angiomatoid Fibrous Histiocytoma: A Series of 21 Cases with Variant Morphology. Int J ClinExpPathol., 8, 772-778.

[8] Sheehan, K.M., Leader, M.B., Sexton, S., Cunningham, F. and Leen, E. (2004) Recurrent Aneurysmal Fibrous Histiocytoma. JClinPathol, 57, 312-313. https://doi.org/10.1136/jcp.2003.010967 